\title{
A Note on Tropical Triangles in the Plane
}

\author{
M. ANSOLA M. J. de la PUENTE \\ Departamento de Algebra Facultad de Matemáticas, Universidad Complutense, Madrid 28040, Spain \\ E-mail:mpuente@mat.ucm.es macarenaa@gmail.com
}

\begin{abstract}
We define transversal tropical triangles (affine and projective) and characterize them via six inequalities to be satisfied by the coordinates of the vertices. We prove that the vertices of a transversal tropical triangle are tropically independent and they tropically span a classical hexagon whose sides have slopes $\infty, 0,1$. Using this classical hexagon, we determine a parameter space for transversal tropical triangles. The coordinates of the vertices of a transversal tropical triangle determine a tropically regular matrix. Triangulations of the tropical plane are obtained.
\end{abstract}

Keywords tropical triangles, tropical triangulation, linear inequalities, convexity, tropical semi-field MR(2000) Subject Classification 52C35, 52C20, 15A39, 12K99

\section{Introduction}

Triangles are, after points and lines, the simplest figures in any geometry. They can be defined either by three different non-collinear points, called vertices, or by three non-concurrent pairwise transversal lines, called sides. The vertices of a triangle span the plane (affine or projective) and they are independent points. Also, triangles are the simplest two-dimensional convex figures and they provide tilings of the plane, or triangulations. This is all elementary mathematics.

If we move to the tropical plane, then natural questions about triangles arise. How are the notions of span, independence, convexity and transversality (in their tropical versions) related to tropical triangles? On the one hand, in [1-2] it is shown that no finite family of points can tropically span the plane. On the other hand, tropical convexity has been thoroughly studied in [3]; in particular, tropical triangles are defined there, and five combinatorial types of tropical triangles are shown to exist, up to symmetry. However, most such tropical triangles have non-transversal sides.

In this paper, we propose a finer definition of tropical triangle. Such tropical triangles will be called transversal. It amounts to restricting to one combinatorial type from [3]. Our starting point (from elementary geometry) is the following: the tropical triangles we are interested in are only those defined by three different non-collinear points $a, b, c$ such that, when joined by pairs there yield three different lines $a b, b c, c a$ which, when intersected by pairs yield the original points. The intersection (resp. join) we are talking about here is stable intersection (resp. stable join) and these are the right notions to consider in tropical geometry. And it turns out that, in transversal conditions stable intersection (resp. stable join) is nothing but plain intersection (resp. join). 
It is a basic fact that a tropical line in the plane carries a special point, called vertex. Therefore, with a tropical triangle $T$ we can associate a family of six points: the three vertices of $T$ and the three vertices of the tropical sides of $T$. In Theorem 3 it is proved that the tropical span of the vertices of a transversal tropical triangle $T$ equals the classical convex hull of the six related points. This means that giving a transversal tropical triangle $T$ amounts to giving a classical hexagon, $\mathcal{H}(T)$, the sides of which have slopes $\infty, 0,1$. It is our opinion that all triangles should look alike, in any geometry and this is not the case for tropical triangles, as defined in [3], but it is certainly true, for transversal tropical triangles. Moreover, the lattice lengths the sides of $\mathcal{H}(T)$ parameterize the tropical triangle $T$.

In [4], Joswig raises the question of what should be the right notion of tropical triangulation. For the tropical plane (affine or projective), we give the following solution: a triangulation $\mathcal{T}$ of the tropical plane is a family of transversal tropical triangles $\left\{T_{j}: j \in J\right\}$ such that the associated family $\left\{\mathcal{H}\left(T_{j}\right): j \in J\right\}$ tessellate the classical plane. More precisely, if two tropical triangles $T_{1}, T_{2}$ in $\mathcal{T}$ meet, all they share is one vertex and one side and, moreover, the associated classical hexagons $\mathcal{H}\left(T_{1}\right), \mathcal{H}\left(T_{2}\right)$ have just one side in common, including the two end points, see Figure 1.

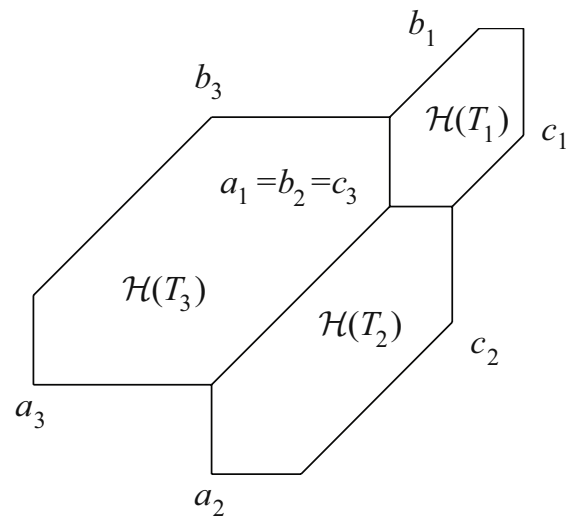

Figure 1 Tropical triangulation of the plane

The main results of the paper are Theorems 1 and 2, where transversal tropical triangles are characterized by six strict inequalities to be satisfied by the coordinates of the vertices (cases affine and projective). The inequalities in Theorem 2 show a high level of symmetry.

We work exclusively in the tropical setting. Some authors solve problems in tropical geometry by the lifting method, see [5-7]. This means that they start with a question in tropical geometry, lift the question to classical geometry (when possible, see [8-9]), solve the question there (when possible) and tropicalize the solution (always possible). Although the existence of liftings is one powerful reason to do tropical geometry, we will not use liftings at all.

Tropical geometry (and the study of certain closely related objects called amoebas) is a very recent trend in Mathematics, see [6,10-18]. Its algebraic counterpart is tropical algebra and it has been studied since about 1950. It is related to control theory, automata theory, scheduling theory, discrete event systems, optimization, combinatorics, mathematical physics, etc. It has applications in complex and real enumerative geometry, phylogenetics, etc., see [19-22]. It is a fast developing geometry, following the track of algebraic geometry, see [23-25]. It is connected 
with toric geometry, see [26-28].

Tropical algebra has appeared in the literature under various denominations such as minimax-algebra, max-algebra, min-algebra, max-plus algebra, min-plus algebra, semirings, moduloïds, diö̈ds, pseudorings, pseudomodules, band spaces over belts, idempotent mathematics (semirings, analysis, calculus, etc.), Maslov dequantization, etc., see [17, 29-35].

As a rule, we will use the adjective classical (for classical mathematics) as opposed to tropical. In this note we present results which can be traced back to [10], but have been very much elaborated afterwards. Transversal triangles are called stable triangles there. A previous version of this paper can be found in ArXiv.

\section{Notations and Background on Elementary Tropical Geometry}

The tropical semi-field is the set $\mathbb{T}:=\mathbb{R} \cup\{-\infty\}$ endowed with tropical addition $\oplus$ and tropical multiplication $\odot$. These operations are defined as follows:

$$
a \oplus b=\max \{a, b\}, \quad a \odot b=a+b,
$$

for $a, b \in \mathbb{R} \cup\{-\infty\}$. Note that tropical addition is idempotent, i.e., $a \oplus a=a$, for $a \in \mathbb{T}$. Tropical addition is associative, commutative and $-\infty$ is the neutral element. Tropical multiplication is associative, commutative and 0 is the neutral element. The element $-a$ is inverse to $a$ with respect to $\odot$, for $a \in \mathbb{R}$. Moreover, multiplication is distributive over addition, since

$$
a+\max \{b, c\}=\max \{a+b, a+c\} .
$$

However, we cannot find an inverse element, with respect to $\oplus$, for any $a \in \mathbb{R}$, and this is why $\mathbb{T}$ is NOT a field.

For $n \in \mathbb{N}$, the tropical affine $n$-space is $\mathbb{T}^{n}$, where tropical addition and multiplication are defined coordinatewise. For addition, the neutral element is $p_{n}:=(-\infty, \ldots,-\infty)$, but one must realize that $a \oplus b=b$ does not imply $a=p_{n}$.

The tropical projective $n$-space, $\mathbb{T P}^{n}$, is the defined as follows. In the space $\mathbb{T}^{n+1} \backslash\left\{p_{n+1}\right\}$ we define an equivalence relation $\sim$ by letting $\left(b_{1}, \ldots, b_{n+1}\right) \sim\left(c_{1}, \ldots, c_{n+1}\right)$ if there exists $\lambda \in \mathbb{R}$ such that $\left(b_{1}+\lambda, \ldots, b_{n+1}+\lambda\right)=\left(c_{1}, \ldots, c_{n+1}\right)$. The equivalence class of $\left(b_{1}, \ldots, b_{n+1}\right)$ is denoted by $\left[b_{1}, \ldots, b_{n+1}\right]$ and its elements are obtained by adding multiples of the vector $(1, \ldots, 1)$ to the point $\left(b_{1}, \ldots, b_{n+1}\right)$.

Points in $\mathbb{T}^{n}$ (resp. $\mathbb{T P}^{n}$ ) without infinite coordinates will be called interior points. The rest of the points will be called boundary points. The boundary of $\mathbb{T}^{n}$ (resp. $\mathbb{T P}^{n}$ ) is the union of its boundary points. Note that $p_{n}$ is a boundary point in $\mathbb{T}^{n}$.

In this paper we only work in the tropical plane (affine or projective). Therefore, we will just present the following notions for $n=2$, although they apply in any dimension $n \geq 2$. We will use $X, Y, Z$ as variables.

The tropical projective plane $\mathbb{T P}^{2}$ is covered by three copies of the tropical affine plane $\mathbb{T}^{2}$ as follows. The maps

$$
\begin{array}{ll}
j_{3}: \mathbb{T}^{2} \rightarrow \mathbb{T P}^{2}, & (x, y) \mapsto[x, y, 0], \\
j_{2}: \mathbb{T}^{2} \rightarrow \mathbb{T P}^{2}, & (x, z) \mapsto[x, 0, z], \\
j_{1}: \mathbb{T}^{2} \rightarrow \mathbb{T P}^{2}, & (y, z) \mapsto[0, y, z]
\end{array}
$$


are injective and we have $\mathbb{T} \mathbb{P}^{2}=\operatorname{im} j_{3} \cup \operatorname{im} j_{2} \cup \operatorname{im} j_{1}$. The complementary set of $\operatorname{im} j_{3}$ is

$$
\mathbb{T P}^{2} \backslash \operatorname{im} j_{3}=\{[x, y,-\infty]: x, y \in \mathbb{T}\}
$$

and it is in bijection with $\mathbb{T P}^{1}$ (forget the last coordinate!), similarly for $j_{k}, k=1,2$. It is easy to check that the set of interior points of $\mathbb{T P}^{2}$ is equal to the intersection $\operatorname{im} j_{3} \cap \operatorname{im} j_{2} \cap \operatorname{im} j_{1}$.

As we already know, the projective tropical coordinates of a point in $\mathbb{T P}^{2}$ are not unique. In order to work with a unique triple of coordinates for (almost) each point, we choose a normalization. A few points in $\mathbb{T P}^{2}$ do not admit normalized coordinates, but this will not be a serious obstacle. Our favorite normalization is making the last coordinate equal to zero. We will express this by saying that we work in $Z=0$; it means passing from the projective plane to the affine one, via $j_{3}$. Of course, the point $[a, b,-\infty]$ does not admit normalized coordinates in $Z=0$, for $a, b \in \mathbb{T}$. Other possible normalizations are $Y=0$, or $X=0$, or $X+Y+Z=0$, etc.

Let $n \in \mathbb{N}$. We can consider tropical polynomials in any number of variables $X_{1}, X_{2}, \ldots, X_{n}$ with coefficients in $\mathbb{T}$. Write $\bar{X}$ for $\left(X_{1}, X_{2}, \ldots, X_{n}\right)$ and let $i=\left(i_{1}, i_{2}, \ldots, i_{n}\right) \in \mathbb{N}^{n}$ be a multiindex. Then write

$$
\bar{X}^{\odot i}=X_{1}^{\odot i_{1}} \odot \cdots \odot X_{n}^{\odot i_{n}}=i_{1} X_{1}+\cdots+i_{n} X_{n}
$$

and let

$$
p(\bar{X})=\bigoplus_{i \in I} a_{i} \odot \bar{X}^{\odot i}=\max _{i \in I}\left\{a_{i}+i_{1} X_{1}+\cdots+i_{n} X_{n}\right\},
$$

where $I \subset \mathbb{N}^{n}$ is some finite set and $a_{i} \in \mathbb{T}$. Being $-\infty$ the neutral element for tropical addition, terms having $a_{i}=-\infty$ may be omitted in $p$. The polynomial $p$ is homogeneous if there exists $d \in \mathbb{N}$ such that $i_{1}+\cdots+i_{n}=d$, for all $i \in I$ with $a_{i} \neq-\infty$. If $p$ is homogeneous and $a_{i} \neq-\infty$, for all

$$
i \in\{(d, 0, \ldots, 0),(0, d, 0, \ldots, 0), \ldots,(0, \ldots, 0, d)\},
$$

then we say that $p$ has degree $d$. By means of an extra variable, we can homogenize a nonhomogeneous tropical polynomial $p$, easily. We will use capital letters to denote homogeneous polynomials and small letters to denote arbitrary polynomials. The non-homogeneous polynomial $p$ is said to have degree $d$ if its homogenization has degree $d$. In particular, the degree is NOT defined for some tropical polynomials. In the literature one can find a more general notion of tropical degree, but this one is good enough for our purposes.

A tropical polynomial $p$ (resp. homogeneous polynomial $P$ ) of degree $d>0$ in $n$ (resp. $n+1$ ) variables defines a so-called tropical affine hypersurface $V(p)$ (resp. projective) hypersurface $V(P)$ in $\mathbb{T}^{n}$ (resp. $\mathbb{T P}^{n}$ ). By definition, $V(p)$ (resp. $\left.V(P)\right)$ is the set of points in $\mathbb{T}^{n}$ (resp. $\mathbb{T P}^{n}$ ) where the maximum is attained, at least, twice. This is certainly different from the classical definition of algebraic hypersurface. A tropical hyperplane is a tropical hypersurface defined by a linear polynomial.

Let $n=2$. In this case, we use variables $X, Y, Z$, instead of $X_{1}, X_{2}, X_{3}$. In the tropical plane, hypersurfaces are called tropical curves. We have tropical lines, conics, cubics, etc., meaning curves defined by tropical polynomials of degree 1, 2, 3, etc., which are homogeneous in the projective case. Every tropical projective curve $\mathcal{C}$ is covered by three associated affine curves, namely, $\mathcal{C}=\mathcal{C}_{3} \cup \mathcal{C}_{2} \cup \mathcal{C}_{1}$, where $\mathcal{C}_{k}:=\mathcal{C} \cap i \operatorname{im} j_{k}, k=1,2,3$. 
The simplest tropical plane curves are lines, of course. A tropical line in the affine plane is $V(p)$, where

$$
p=a \odot X \oplus b \odot Y \oplus c=\max \{a+X, b+Y, c\}
$$

for some coefficients $a, b, c \in \mathbb{R}$. Notice that the point $p_{2}=(-\infty,-\infty) \in \mathbb{T}^{2}$ does not belong to $V(p)$. The line $L:=V(p)$ is easy to describe. The points $(c-a, c-b),(-\infty, c-b)$ and $(c-a,-\infty)$ belong to $L$; the first one is interior, while the other two are boundary points. Moreover, $L$ is the union of three rays, meeting at the interior point $(c-a, c-b)$. The directions of these rays are West, South and North-East. Notice that there is no boundary point at the end of the North-East ray of $L$. The homogenization of $p$ is

$$
P=a \odot X \oplus b \odot Y \oplus c \odot Z=\max \{a+X, b+Y, c+Z\} .
$$

$P$ defines the tropical line $\bar{L}:=V(P)$ in $\mathbb{T P}^{2}$ and $L$ embeds in $\bar{L}$ via $j_{3}$. In particular, we have

$$
j_{3}(c-a, c-b)=[c-a, c-b, 0]=[-a,-b,-c],
$$

and this point is called the vertex of $\bar{L}$. We also have

$$
j_{3}(c-a,-\infty)=[-a,-\infty,-c], \quad j_{3}(-\infty, c-b)=[-\infty,-b,-c],
$$

and we find that $[-a,-b,-\infty]$ is the only point in $\bar{L} \backslash L$. Actually, this is the missing boundary point in the North-East ray of $L$. In addition, the identification of $L$ with its image in $\bar{L}$ via $j_{3}$ allows us to have a graphical representation of $\bar{L}$ in $Z=0$, where the only missing point is $[-a,-b,-\infty]$. Of course, we can also represent $\bar{L}$ in $Y=0$ or in $X=0$, easily.

Let $n \in \mathbb{N}$. Given an $n \times n$ matrix $A$ with real entries, the tropical determinant of $A$, (also called permanent) is defined as follows:

$$
|A|_{\text {trop }}:=\left|\begin{array}{ccc}
a_{11} & \cdots & a_{1 n} \\
\vdots & & \vdots \\
a_{n 1} & \cdots & a_{n n}
\end{array}\right|_{\text {trop }}=\max _{\sigma \in S_{n}}\left\{a_{1 \sigma(1)}+\cdots+a_{n \sigma(n)}\right\}
$$

where $S_{n}$ denotes the permutation group in $n$ symbols. The matrix $A$ is tropically singular if the maximum in $|A|_{\text {trop }}$ is attained, at least, twice. Otherwise, $A$ is tropically regular, (also said that $A$ has a strong permanent).

There exists a duality between lines in the tropical projective plane and interior points in the projective plane. Given an interior point $a=\left[a_{1}, a_{2}, a_{3}\right]$ in $\mathbb{T P}^{2}$, let $L_{a}$ denote the line in $\mathbb{T P}^{2}$ defined by tropical linear form to be $a_{1} \odot X \oplus a_{2} \odot Y \oplus a_{3} \odot Z$. Obviously, we have

$$
b \in L_{a} \Longleftrightarrow a \in L_{b}
$$

meaning that $\max \left\{a_{1}+b_{1}, a_{2}+b_{2}, a_{3}+b_{3}\right\}$ is attained, at least, twice.

Let two points $a, b$ in the tropical plane (affine or projective) be given. If $a$ and $b$ do not both lie on a classical line of slope $\infty, 0,1$, then there exists a unique tropical line through both points and this line is called the (tropical) join of $a$ and $b$. Otherwise, there exist infinitely many tropical lines going through $a$ and $b$. The (tropical) stable join of $a, b$ is defined as the limit, as $\epsilon$ tends to zero, of the tropical lines going through perturbed points $a^{v_{\epsilon}}, b^{v_{\epsilon}}$. Here, $a^{v_{\epsilon}}$ denotes a translation of $a$ by a length- $\epsilon$ vector $v_{\epsilon}$, see $[6,12]$. We denote this line by $a b$. Of 
course, if $a$ and $b$ do not both lie on a classical line of slope $\infty, 0,1$, then their join and their stable join coincide.

Now, the intersection of two tropical lines $L, M$ in the plane (affine or projective) may be a point or a ray. In the former case, we will say that the tropical lines $L$ and $M$ are transversal. In any case, we define the so-called stable intersection, $L \cap_{\text {est }} M$, as the limit point, as $\epsilon$ tends to zero, of the intersection of perturbed lines $L^{v_{\epsilon}}, M^{v_{\epsilon}}$. Here, $L^{v_{\epsilon}}$ denotes a translation of $L$ by a length $-\epsilon$ vector $v_{\epsilon}$.

It is well known that duality transforms stable join into stable intersection and conversely, i.e.,

$$
L_{a} \cap_{\mathrm{est}} L_{b}=c \Longleftrightarrow a b=L_{c}
$$

for $a, b, c$ interior points in $\mathbb{T P}^{2}$. By duality, we will say that the points $a, b$ are transversal if there exists a unique tropical line passing $a$ and $b$.

Stable intersection and stable join are defined in wider generality. Now consider $n$ hyperplanes in $\mathbb{T P}^{n}$ and take an associated system of $n$ linear tropical homogeneous polynomials in $n+1$ variables. Let $A$ be the $n \times(n+1)$ coefficient matrix of the system. For each $j=1, \ldots, n+1$, let $A^{j}$ be the square matrix obtained by deleting the $j$-th column from $A$. Then the tropical version of Cramer's rule tells us that the point $\left[\left|A^{1}\right|_{\text {trop }}, \ldots,\left|A^{n+1}\right|_{\text {trop }}\right] \in \mathbb{T P}^{n}$ is the stable intersection of the $n$ given hyperplanes. Moreover, the intersection of the $n$ hyperplanes equals the stable intersection if and only if $A^{j}$ is tropically regular, for all $j=1, \ldots, n+1$; see [6].

For tropical lines in the plane, the tropical version of Cramer's rule goes as follows: the stable intersection of the lines $L_{a}$ and $L_{b}$ is the point

$$
\left[\max \left\{a_{2}+b_{3}, b_{2}+a_{3}\right\}, \max \left\{a_{1}+b_{3}, b_{1}+a_{3}\right\}, \max \left\{a_{1}+b_{2}, b_{1}+a_{2}\right\}\right] .
$$

Since the computation of this point is nothing but a tropical version of the cross-product of the triples $a$ and $b$, we will denote it by $a \otimes b$. Notice that $a \otimes b=b \otimes a$. In other words, the tropical version of Cramer's rule in the plane means

$$
L_{a} \cap_{\mathrm{est}} L_{b}=a \otimes b,
$$

and, by duality,

$$
a b=L_{a \otimes b} .
$$

In particular, the vertex of the tropical line $a b$ is the point $-(a \otimes b)$.

Tropical cross-product satisfies $a \otimes a=-a$, if $a$ is interior. But, unluckily, tropical crossproduct is non-associative and so, it seems tricky to compute expressions such as $(c \otimes a) \otimes(a \otimes b)$. However, we will see in Corollary 1 that only three values are possible for this long expression.

Lemma 1 Interior points $a, b \in \mathbb{T P}^{2}$ are transversal if and only if $a \otimes b \notin\{-a,-b\}$.

Proof If $a, b$ are interior points in $\mathbb{T}^{2}$, then a simple computation shows that $a \otimes b \in\{-a,-b\}$ if and only if the points $a, b$ lie on a classical line of slope $\infty, 0,1$, and this is the non-transversal case. Now if $a, b$ are interior points in $\mathbb{T P}^{2}$, we obtain the desired result, either passing to the affine setting, or using Cramer's rule. Indeed, an easy computation shows that $a \otimes b \in\{-a,-b\}$ if and only if some coordinate in $a \otimes b=\left[\max \left\{a_{2}+b_{3}, b_{2}+a_{3}\right\}, \max \left\{a_{1}+b_{3}, b_{1}+a_{3}\right\}, \max \left\{a_{1}+\right.\right.$ $\left.\left.b_{2}, b_{1}+a_{2}\right\}\right]$ is tropically singular. 
Lemma 2 Suppose that $a, b, c$ are three interior points in the tropical plane (affine or projective) which are tropically non-collinear. Assume that $a, b$ are transversal, $a, c$ are transversal and the tropical lines $a b, c a$ are transversal. Then $(c \otimes a) \otimes(a \otimes b)=a$, i.e., $c a \cap_{\mathrm{est}} a b=c a \cap a b=a$.

Proof Since the lines $a b$ and $c a$ are transversal, then the points $c \otimes a, a \otimes b$ do not lie on a classical line of slope $\infty, 0,1$. Since the points $a, b$ are transversal, then $a$ and $b$ lie on different rays of the tropical line with vertex at $-(a \otimes b)$. A similar situation is true for the points $a, c$ and the tropical line with vertex at $-(c \otimes a)$. Then $a$ is the unique point of intersection of $a b$ and $c a$. This gives a picture in $\mathbb{R}^{2}$. A symmetric picture, with respect to the origin, is obtained by considering the points $c \otimes a, a \otimes b$ and the vertex, $-((c \otimes a) \otimes(a \otimes b))$, of the unique tropical line through them. Therefore $(c \otimes a) \otimes(a \otimes b)=a$.

The corollary below is a direct consequence of Lemmas 1 and 2 .

Corollary 1 Suppose that $a, b, c$ are three interior points in the tropical plane (affine or projective) which are tropically non-collinear. Then

$$
(c \otimes a) \otimes(a \otimes b) \in\{-(c \otimes a),-(a \otimes b), a\} .
$$

\section{Transversal Triangles in Tropical Plane}

In this section, $a, b, c$ will always denote three different interior points in the tropical plane (affine or projective) which are tropically non-collinear. The tropical sides defined by $a, b, c$ are the tropical lines $a b, b c, c a$ and we know that the points $-(a \otimes b),-(b \otimes c)$ and $-(c \otimes a)$ are the vertices of them.

In the first example below, we see that $a b \cap_{\text {est }} b c \neq b$ and this is unpleasant for a triangle. In the second one, we see have $c a \cap_{\text {est }} a b=a, a b \cap_{\text {est }} b c=b, b c \cap_{\text {est }} c a=c$, showing the kind of triangles we are interested in.

Example 1 In $\mathbb{T P}^{2}$ take $a=[-1,1,0], b=[0,0,0], c=[-1,2,0]$. Then, $a \otimes b=[1,0,1]$ and $b \otimes c=[2,0,2]$, so that $(a \otimes b) \otimes(b \otimes c)=[2,3,2]=-(a \otimes b) \neq b$.

Example 2 The reader can easily check that the points $a=[-3,-1,0], b=[0,0,0], c=$ $[-1,2,0]$ satisfy $(c \otimes a) \otimes(a \otimes b)=a,(a \otimes b) \otimes(b \otimes c)=b$ and $(b \otimes c) \otimes(c \otimes a)=c$.

Thus, for some purposes, some care must be taken in order to define triangles in the tropical plane (affine or projective).

Definition 1 Three points a, b, c define a transversal tropical triangle abc if the vertices $a, b, c$ are pairwise transversal and so are the tropical sides.

Definition 2 Three points a,b, c define a good tropical triangle abc if, by stable join, they give rise to three tropical lines $a b, b c, c a$ which, stably intersected by pairs, yield the original points $a, b, c$, i.e., $c a \cap_{\mathrm{est}} a b=a, a b \cap_{\mathrm{est}} b c=b, b c \cap_{\mathrm{est}} c a=c$.

Definition 3 Three points $a, b, c$ define a proper tropical triangle abc if $a, b, c,-(a \otimes b),-(b \otimes c)$ and $-(c \otimes a)$ are six different points. A tropical triangle which is not proper will be called improper.

Example 3 The points $[0,0,0],[1,1,0],[0,1,0]$ define a good tropical triangle and so do the points $[0,0,0],[1,1,0],[1,0,0]$. Both triangles are improper. The triangle in Example 2 is good and proper. 
By Lemmas 1 and 2 and Corollary 1, a tropical triangle $T$ is transversal if and only if $T$ is good and proper.

Theorem 1 Three points $a=\left(a_{1}, a_{2}\right), b=\left(b_{1}, b_{2}\right), c=\left(c_{1}, c_{2}\right)$ in $\mathbb{R}^{2}$ determine a transversal tropical triangle if and only if, perhaps after relabeling, the following inequalities hold:

$$
\begin{aligned}
& a_{1}<b_{1}<c_{1}, a_{2}<c_{2}<b_{2}, \\
& b_{1}-b_{2}<a_{1}-a_{2}<c_{1}-c_{2} .
\end{aligned}
$$

In particular, these inequalities determine an open unbounded polyhedron in $\mathbb{R}^{6}$, which can be viewed projectively in $\mathbb{T P}^{5}$.

Proof The inequalities hold for $\left(a_{1}, a_{2}, b_{1}, b_{2}, c_{1}, c_{2}\right) \in \mathbb{R}^{6}$ if and only if they hold for $\left(a_{1}+\right.$ $\left.\lambda, a_{2}+\lambda, b_{1}+\lambda, b_{2}+\lambda, c_{1}+\lambda, c_{2}+\lambda\right)$, for $\lambda \in \mathbb{R}$. This proves the last statement.

Let us assume that the six inequalities hold. Easy computations yield $a \otimes b=\left[b_{2}, b_{1}, a_{1}+b_{2}\right]$, $b \otimes c=\left[b_{2}, c_{1}, c_{1}+b_{2}\right]$ and $c \otimes a=\left[c_{2}, c_{1}, c_{1}+a_{2}\right]$. Then we obtain $(c \otimes a) \otimes(a \otimes b)=$ $\left[b_{2}+a_{1}+c_{1}, c_{1}+a_{2}+b_{2}, b_{2}+c_{1}\right]=a$ and, similarly, $(a \otimes b) \otimes(b \otimes c)=b$ and $(b \otimes c) \otimes(c \otimes a)=c$, proving that the tropical triangle $a b c$ is transversal.

Conversely, suppose that the points $a, c, b$ define a transversal triangle. Then no two of the given points lie on a classical line of slope $\infty, 0,1$. In particular, the numbers $a_{1}-a_{2}, b_{1}-b_{2}$, $c_{1}-c_{2}$ are pairwise different. We may assume that

$$
\begin{aligned}
& a_{1}<\min \left\{b_{1}, c_{1}\right\}, \\
& b_{1}-b_{2}<c_{1}-c_{2} .
\end{aligned}
$$

Then we compute the point $(c \otimes a) \otimes(a \otimes b)$ and see that one of its coordinates is given by the value of a singular tropical determinant, unless

$$
b_{1}-b_{2}<a_{1}-a_{2}<c_{1}-c_{2} .
$$

Now classical and tropical geometry tell us that the coordinates of the vertex $-(a \otimes b)$ are $\left[a_{1}, a_{1}-b_{1}+b_{2}, 0\right]$, on the one hand, and $\left[-\max \left\{a_{2}, b_{2}\right\},-b_{1},-a_{1}-b_{2}\right]$ on the other. Equating these projective tropical coordinates yields

$$
\max \left\{a_{2}, b_{2}\right\}=b_{2} .
$$

A similar computation for $-(c \otimes a)$ yields

$$
\max \left\{a_{2}, c_{2}\right\}=c_{2} .
$$

Therefore, we have

$$
a_{2}<\min \left\{b_{2}, c_{2}\right\} .
$$

We proceed to determine the values of $\min \left\{b_{1}, c_{1}\right\}$ and $\min \left\{b_{2}, c_{2}\right\}$. There are only three possible cases, because the condition $b_{1}-b_{2}<c_{1}-c_{2}$ eliminates the possibility $b_{1}>c_{1}$ and $b_{2}<c_{2}$. Now if $b_{1}>c_{1}$ and $b_{2}>c_{2}$, then $b \otimes c=\left[b_{2}, b_{1}, c_{1}+b_{2}\right]$ and $a \otimes b=\left[b_{2}, b_{1}, a_{1}+b_{2}\right]$, so that $(b \otimes c) \otimes(a \otimes b)=-(b \otimes c) \neq b$, contradicting transversality. And if $b_{1}<c_{1}$ and $b_{2}<c_{2}$, then $b \otimes c=\left[c_{2}, c_{1}, c_{1}+b_{2}\right]$ and $c \otimes a=\left[c_{2}, c_{1}, c_{1}+a_{2}\right]$ so that $(c \otimes a) \otimes(b \otimes c)=-(b \otimes c) \neq c$, contradicting transversality. Therefore, $b_{1}<c_{1}$ and $b_{2}>c_{2}$ and all six inequalities have been proved. 


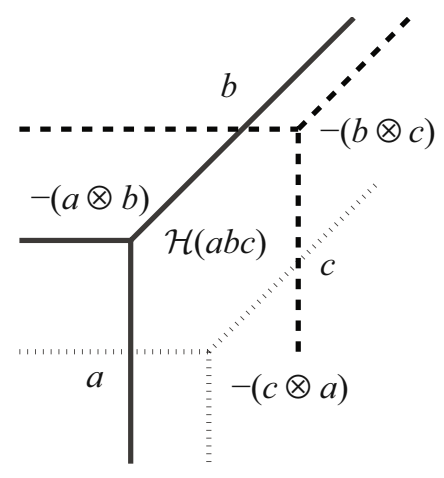

Figure 2 Transversal tropical triangle

Let us make a picture of a tropical triangle $a b c$ in $\mathbb{R}^{2}$, say in $Z=0$. The six inequalities shown in the previous theorem must be satisfied. We have to represent the vertices $a, b, c$ and the tropical sides $a b, b c, c a$, whose vertices are the points

$$
-(a \otimes b)=\left(a_{1}, a_{1}+b_{2}-b_{1}\right), \quad-(c \otimes a)=\left(a_{2}+c_{1}-c_{2}, a_{2}\right), \quad-(b \otimes c)=\left(c_{1}, b_{2}\right) .
$$

Then we obtain a classical convex hexagon having vertices (in clockwise order)

$$
a,-(a \otimes b), b,-(b \otimes c), c,-(c \otimes a)
$$

and slopes $\infty, 1,0, \infty, 1,0$, see Figure 2 . It will be denoted by $\mathcal{H}(a b c)$. The six inequalities shown in Theorem 1 provide the lengths of the sides of $\mathcal{H}(a b c)$.

Up to translation, scaling and exchange of variables $X, Y, Z$, a transversal tropical triangle $T$ is determined by a classical convex hexagon $\mathcal{H} \subset \mathbb{R}^{2}$ of slopes $\infty, 0,1$. Now, $\mathcal{H}$ is determined by the lattice lengths of its sides, which are real positive numbers $l_{1}, l_{2}, \ldots, l_{6}$ such that

$$
l_{j-2}+l_{j-1}=l_{j+1}+l_{j+2}, \quad j=1,2(\text { or } j=1,2, \ldots, 6),
$$

where subscripts are taken modulo 6 . Therefore, the set

$$
P:=\left\{\left[l_{1}, l_{2}, \ldots, l_{6}\right] \in \mathbb{T P}^{5}: l_{j}>0 \text { and } l_{j-2} \odot l_{j-1}=l_{j+1} \odot l_{j+2}, j=1,2\right\}
$$

is a parameter space for transversal tropical triangles. The dimension of $P$ is three and any positive numbers $l_{1}, l_{2}, l_{3}, l_{5} \in \mathbb{R}$ such that

$$
l_{5}<\min \left\{l_{1}+l_{2}, l_{2}+l_{3}\right\}
$$

determine a unique point in $P$.

Let us now translate the six inequalities found in Theorem 1 to the projective setting.

Theorem 2 Let $a=\left[a_{1}^{\prime}, a_{2}^{\prime}, a_{3}^{\prime}\right], b=\left[b_{1}^{\prime}, b_{2}^{\prime}, b_{3}^{\prime}\right], c=\left[c_{1}^{\prime}, c_{2}^{\prime}, c_{3}^{\prime}\right] \in \mathbb{T P}^{2}$ be three different interior points. Then $a, b, c$ determine a transversal tropical triangle if and only if, perhaps after relabeling, the following six inequalities hold:

$$
\begin{aligned}
& b_{1}^{\prime}-b_{2}^{\prime}<a_{1}^{\prime}-a_{2}^{\prime}<c_{1}^{\prime}-c_{2}^{\prime}, \\
& a_{2}^{\prime}-a_{3}^{\prime}<c_{2}^{\prime}-c_{3}^{\prime}<b_{2}^{\prime}-b_{3}^{\prime}, \\
& c_{3}^{\prime}-c_{1}^{\prime}<b_{3}^{\prime}-b_{1}^{\prime}<a_{3}^{\prime}-a_{1}^{\prime} .
\end{aligned}
$$


Proof Without loss of generality, we may work in $Z=0$. Then $a=\left[a_{1}^{\prime}-a_{3}^{\prime}, a_{2}^{\prime}-a_{3}^{\prime}, 0\right]$, $b=\left[b_{1}^{\prime}-b_{3}^{\prime}, b_{2}^{\prime}-b_{3}^{\prime}, 0\right], c=\left[c_{1}^{\prime}-c_{3}^{\prime}, c_{2}^{\prime}-c_{3}^{\prime}, 0\right]$. Now, notice that the points $\left(a_{1}^{\prime}-a_{3}^{\prime}, a_{2}^{\prime}-a_{3}^{\prime}\right)$, $\left(b_{1}^{\prime}-b_{3}^{\prime}, b_{2}^{\prime}-b_{3}^{\prime}\right),\left(c_{1}^{\prime}-c_{3}^{\prime}, c_{2}^{\prime}-c_{3}^{\prime}\right)$ belong to $\mathbb{R}^{2}$ and satisfy the six inequalities shown in Theorem 1 , where $a_{j}=a_{j}^{\prime}-a_{3}^{\prime}, b_{j}=b_{j}^{\prime}-b_{3}^{\prime}, c_{j}=c_{j}^{\prime}-c_{3}^{\prime}, j=1,2$, and so we are done.

Notice the high cyclic symmetry shown in the six inequalities in Theorem 2. Notice also that the letters $a, b, c$ are arranged as a latin square.

Now we proceed to relate tropical transversal triangles with the notions of tropical span, tropical independence and strong permanent. The following definitions are standard.

Definition 4 In $\mathbb{T}^{n}$ or $\mathbb{T P}^{n}$, let $u_{1}, \ldots, u_{s}$ be different interior points. A point $u$ is tropically spanned by $u_{1}, \ldots, u_{s}$ if it can be written as

$$
u=\lambda_{1} \odot u_{1} \oplus \cdots \oplus \lambda_{s} \odot u_{s}=\max \left\{\lambda_{1}+u_{1}, \ldots, \lambda_{s}+u_{s}\right\},
$$

for some $\lambda_{1}, \ldots, \lambda_{s} \in \mathbb{T}$, and not all $\lambda_{j}$ equal to $-\infty$.

The points $u_{1}, \ldots, u_{s}$ are tropically independent if there does not exist $j \in\{1, \ldots, s\}$ such that $u_{j}$ is tropically spanned by $u_{1}, \ldots, u_{j-1}, u_{j+1}, \ldots, u_{s}$.

Notice that all points spanned by interior points are interior.

Tropical span is closer to classical convexity than to linear, affine or projective span. This is shown in the following theorem, which can be traced back to $[1-4,35]$. The classical segment defined by points $a, b$ will be denoted $\operatorname{conv}(a, b)$.

Theorem 3 Let three points $a, b, c$ in $\mathbb{T P}^{2}$ determine a transversal tropical triangle. Then the following assertions hold.

1 The points tropically spanned by $a, c, b$ are exactly those of the solid hexagon $\mathcal{H}(a b c)$.

2 The points $a, b, c$ are tropically independent.

3 The matrix $3 \times 3$ given by coordinates of $a, b, c$ is tropically regular (i.e., this matrix has a strong permanent).

Proof We may work in $Z=0$ and therefore, we may assume, without loss of generality, that the coordinates of $a, b, c$ satisfy the six inequalities shown in Theorem 1 .

First, we show that the vertices $b, c$ tropically span the union of the classical segments $\operatorname{conv}(b,-(b \otimes c))$ and $\operatorname{conv}(-(b \otimes c), c)$. We have $b=\left[b_{1}, b_{2}, 0\right], c=\left[c_{1}, c_{2}, 0\right]$ with $b_{1}<c_{1}$ and $c_{2}<b_{2}$. Then $-(b \otimes c)=\left[c_{1}, b_{2}, 0\right]=b \oplus c$. A point $u$ tropically spanned by $b, c$ is $u=\lambda \odot b \oplus \mu \odot c$. Working in the projective plane, we may assume $\lambda=0$ and $\mu \in \mathbb{T}$. Thus

$$
u=\left[\max \left\{b_{1}, \mu+c_{1}\right\}, \max \left\{b_{2}, \mu+c_{2}\right\}, \max \{0, \mu\}\right] .
$$

Now, if $0 \geq \mu$, then $b_{2}>\mu+c_{2}$.

- If $b_{1} \geq \mu+c_{1}$, then $u=b$.

- If $b_{1} \leq \mu+c_{1}$, then $u=\left[\mu+c_{1}, b_{2}, 0\right]$ and the point $\left(\mu+c_{1}, b_{2}\right) \in \mathbb{R}^{2}$ runs through the segment $\operatorname{conv}(b,-(b \otimes c))$.

Now, if $0 \leq \mu$, then $b_{1}<\mu+c_{1}$.

- If $b_{2} \geq \mu+c_{2}$, then $u=\left[\mu+c_{1}, b_{2}, \mu\right]=\left[c_{1}, b_{2}-\mu, 0\right]$ and the point $\left(c_{1}, b_{2}-\mu\right) \in \mathbb{R}^{2}$ runs through the segment $\operatorname{conv}(-(b \otimes c), c)$.

- If $b_{2} \leq \mu+c_{2}$, then $u=c$. 
In particular, we have proved that the point $u=b \oplus \mu \odot c \in\{b, c\}$, whenever $\mu$ does not belong to the closed interval $\left[b_{1}-c_{1}, b_{2}-c_{2}\right]$. As a by-product, we have proved that $a$ is not tropically spanned by $b, c$.

In a similar manner, the vertices $a, c$ tropically span the set $\operatorname{conv}(a,-(c \otimes a)) \cup \operatorname{conv}(-(c \otimes$ $a), c)$ and $a, b$ tropically span the set $\operatorname{conv}(a,-(a \otimes b)) \cup \operatorname{conv}(-(a \otimes b), b)$. Therefore, all points on the sides of the classical hexagon $\mathcal{H}(a b c)$ are tropically spanned by $a, b, c$. And, in particular, the tropical independence of $a, b, c$ follows.

Now we consider a point $t=\left(t_{1}, t_{2}\right) \in \mathbb{R}^{2}$ in the interior of the hexagon $\mathcal{H}(a b c)$. Then we take the (unique) point $u=\left(u_{1}, t_{2}\right) \in \mathbb{R}^{2}$ on the border of $\mathcal{H}(a b c)$ with $u_{1}<t_{1}$ and the (unique) point $q=\left(t_{1}, q_{2}\right)$ on the border with $q_{2}<t_{2}$. Then $t=u \oplus q$ and, since $u$ is tropically spanned by $a, b$ and $q$ is tropically spanned by $a, c$, then $t$ is tropically spanned by $a, b, c$.

To finish up, let

$$
A=\left(\begin{array}{ccc}
a_{1} & a_{2} & 0 \\
b_{1} & b_{2} & 0 \\
c_{1} & c_{2} & 0
\end{array}\right)
$$

and check that $|A|_{\text {trop }}=c_{1}+b_{2}$, using the six inequalities. Moreover, the maximum is attained only once, showing that $A$ is tropically regular.

The converse to the second and third statements in Theorem 3 do not hold.

Example 4 The points $a=[0,0,0], b=[3,9,0]$ and $c=[2,1,0]$ are tropically independent but they only satisfy five of the six inequalities in Theorem 1. Moreover, the coordinate matrix $A$ is tropically regular and $|A|_{\text {trop }}=11$.

Now let $T$ be a transversal (i.e., good and proper) tropical triangle $a b c$ (affine or projective). Good improper tropical triangles arise from $T$, by letting two or more adjacent vertices $a,-(a \otimes$ $b), b,-(b \otimes c), c,-(c \otimes a)$ of $\mathcal{H}(a b c)$ collapse, but keeping $a, b, c$ pairwise different. This means that the classical hexagon $\mathcal{H}(a b c)$ collapses to a $n$-polygon, with $3 \leq n<6$ sides (of slopes $\infty, 0,1$ ). Equivalently, at most three inequalities in Theorems 1 or 2 become equalities. The reader can easily sketch (say in $Z=0$ ), the 14 existing combinatorial types of improper good tropical triangles thus obtained. He/she can also arrange them into a graph. The vertices in this graph are improper good tropical triangles and two such triangles $T^{\prime}$ and $T^{\prime \prime}$ in this graph are joined by an edge if $\mathcal{H}\left(T^{\prime \prime}\right)$ is obtained from $\mathcal{H}\left(T^{\prime}\right)$ by collapsing two consecutive vertices. The leaves in this graph correspond either to tropical triangles $T$ such that either $\mathcal{H}(T)$ is a classical pentagon (there are six such leaves) or $\mathcal{H}(T)$ is a classical triangle (there are two such leaves: $T=a b c$ such that $a=-(a \otimes b), b=-(b \otimes c), c=-(c \otimes a)$ or $b=-(a \otimes b), b=-(b \otimes c), a=-(c \otimes a))$. The latter are, by the way, like the two triangles shown in Example 3.

Acknowledgements The authors thank Bertrand for his excellent introductory talks about Tropical Geometry, held in the academic course 2004-05 at Departamento de Algebra, Facultad de Matemáticas, Universidad Complutense de Madrid (Spain) and to Tabera for his interest.

\section{References}

[1] Cuninghame-Green, R. A., Butkovič, P.: Bases in max-algebra. Linear Algebra Appl., 389, 107-120 (2004)

[2] Wagneur, E.: Modulö̈ds and pseudomodules. Dimension theory. Discr. Math., 98, 57-73 (1991)

[3] Develin, M., Sturmfels, B.: Tropical convexity. Doc. Math., 9, 1-27 (2004); Erratum in Doc. Math. 9, 205-206 (2004) 
[4] Joswig, M.: Tropical halfspaces, in J. E. Goodman, J. Pach and E. Welzl (eds.), Combinatorial and Computational Geometry, Cambridge University Press, 2005, MSRI Publications 52, 409-431

[5] Izhakian, Z.: Duality of tropical curves. arXiv:math/0503691 (2005)

[6] Richter-Gebert, J., Sturmfels, B., Theobald, T.: First steps in tropical geometry. in: Idempotent Mathematics and Mathematical Physics, Proceedings Vienna 2003, Litvinov, G. L., Maslov, V. P. (eds.), American Mathematical Society, Contemp. Math., 377, 2005, 289-317

[7] Tabera, L. F.: Tropical constructive Pappus's theorem. IMRN, 39, 2373-2389 (2005)

[8] Jensen, A. N., Markwig, H., Markwig, T.: An algorithm for lifting points in a tropical variety. Collect. Math., 59, 129-165 (2008)

[9] Tabera, L. F.: Tropical plane geometric constructions: a transfer technique in tropical geometry. arXiv: math/0511713 (2007)

[10] Ansola, M.: Propiedades métricas de las cónicas tropicales, Trabajo de investigación DEA, Facultad de Matemáticas, UCM, 2006

[11] Einsiedler, M., Kapranov, M., Lind, D.: Non-archimedean amoebas and tropical varieties. J. Reine Angew. Math., 601, 139-157 (2006)

[12] Gathmann, A.: Tropical algebraic geometry. Jahresbericht der DMV, 108(1), 3-32 (2006)

[13] Gelfand, I. M, Kapranov, M. M., Zelevinsky, A. V.: Discriminants, Resultants and Multidimensional Determinants, Birkhäuser, 1994

[14] Itenberg, I., Mikhalkin, G., Shustin, E.: Tropical Algebraic Geometry, Birkhäuser, 2007

[15] Mikhalkin, G.: What is a tropical curve? Notices AMS, 511-513 (2007)

[16] Sturmfels, B.: Solving Systems of Polynomial Equations, CBMS Regional Conference Series in Math., 97, AMS, Providence, RI, 2002

[17] Viro, O.: Dequantization of real algebraic geometry on logarithmic paper, European Congress of Mathematics, Vol. I (Barcelona 2000); Prog. Math., Vol. 201, Birkhäuser, 2001, 135-146

[18] Viro, O.: What is an amoeba? Notices AMS, 2002, 916-917

[19] Gathmann, A., Markwig, H.: The numbers of tropical plane curves thorugh points in general position. J. Reine Angew. Math., 602, 155-177 (2007)

[20] Mikhalkin, G.: Enumerative tropical algebraic geometry in $\mathbb{R}^{2}$. J. Amer. Math. Soc., 18(2), 313-378 (2005)

[21] Mikhalkin, G.: Tropical geometry and its applications. in Invited lectures, Vol. II, Proceedings of the ICM, Madrid, 2006, M. Sanz-Solé et al. eds., 827-852

[22] Shustin, E.: Patchworking singular algebraic curves, non-archimedean amoebas and enumerative geometry. Algebra i Analiz, 17(2), 170-214 (2005)

[23] Mikhalkin, G., Zharkov, I.: Tropical curves, their jacobians and theta functions. arXiv:math/0612267 (2007)

[24] Speyer, D., Sturmfels, B.: The tropical grassmannian. Adv. Geom., 4, 389-411 (2004)

[25] Vigeland, M. D.: The group law on a tropical elliptic curve. Math. Scand., 104(2), 188-204 (2009)

[26] Hsie, B. Y., Liang, Z. B.: Toric rigid spaces. Acta Mathematica Sinica, English Series, 23(9), 1621-1628 (2007)

[27] Nishinou, T., Siebert, B.: Toric degenerations of toric varieties and tropical curves. Duke Math. J., 135(1), $1-51(2006)$

[28] Shustin, E.: A tropical calculation of the Welschinger invariants of real toric Del Pezzo surfaces. J. Algebraic Geom., 15(2), 285-322 (2006)

[29] Akian, M., Bapat, R., Gaubert, S.: Max-plus Algebra, in L. Hogben, R. Brualdi, A. Greenbaum and R. Mathias (eds.), Handbook of Linear Algebra, Chapman and Hall, 2006

[30] Baccelli, F. L., Cohen, G., Olsder, G. J., Quadrat, J. P.: Syncronization and Linearity, John Wiley, 1992

[31] Butkovič, P.: Max-algebra: the linear algebra of combinatorics? Linear Algebra Appl., 367, 313-335 (2003)

[32] Cuninghame-Green, R.: Minimax Algebra, LNEMS, 166, Springer, 1970

[33] Gaubert, S., Max Plus: Methods and applications of $(\max ,+)$ linear algebra, in R. Reischuk and M. Morvan, (eds.), STACS'97, 1200 in LNCS, 261-282, Lübeck, March 1997, Springer

[34] Litvinov, G. L.: The Maslov dequantization, idempotent and tropical mathematics: a very brief introduction, in: Idempotent Mathematics and Mathematical Physics, Proceedings Vienna 2003, Litvinov, G. L., Maslov, V. P. (eds.), American Mathematical Society, Contemp. Math., 377, 2005, 1-17

[35] Wagneur, E.: Finitely Generated Moduloïds, The Existence and Unicity Problem for Bases, in LNCIS 111, Springer, 1988

[36] Ansola, M., de la Puente, M. J.: Tropical conics for the layman, arXiv:math/0702143v3, to appear in Idempotent Mathematics and Mathematical Physics, Vol. II, Proceedings Moscow 2007, G. L. Litvinov, V. P. Maslov, S. Sergeev (eds.), arXiv:math/ 0709.4119

[37] Litvinov, G. L., Maslov, V. P. (eds.): Idempotent Mathematics and Mathematical Physics, Proceedings Vienna 2003, American Mathematical Society, Contemp. Math., 377, 2005 